\title{
Hindered Rotation Leading to Nonequivalence in Cobaloximes
}

\author{
Debaprasad Mandal, and B. D. Gupta* \\ Department of Chemistry, Indian Institute of Technology, Kanpur, 208 016, India, \\ Tel: +91-512-2597046; Fax: +91-512-2597436; Email: bdg@iitk.ac.in
}

\section{Supporting Information}

Synthesis: Except 2-Me- $\mathrm{C}_{6} \mathrm{H}_{4}-\mathrm{CH}_{2} \mathrm{Co}(\mathrm{dmgH})_{2} \mathrm{Py}$, all compounds are new and they are synthesized by the oxidative addition of $\mathrm{Co}(\mathrm{I})$ to the corresponding benzyl halides. In a typical procedure, $\mathrm{N}_{2}$ gas was bubbled for $10 \mathrm{~min}$ with stirring through a suspension of $\mathrm{ClCo}(\mathrm{dmgH})_{2} \mathrm{Py}(2.02 \mathrm{~g}, 5 \mathrm{mmol})$ in methanol $(30 \mathrm{~mL})$ at $0{ }^{\circ} \mathrm{C}$. The solution turned blue after the addition of a few drops of aqueous $\mathrm{NaOH}$ followed by a $\mathrm{N}_{2}$ purged aqueous solution of $\mathrm{NaBH}_{4}(0.28 \mathrm{~g}, 7.5 \mathrm{mmol}$ in $1 \mathrm{~mL}$ water $)$. The solution immediately turned orange on the addition of an $\mathrm{N}_{2}$ purged solution of appropriate benzyl halide (1.5 equiv.) in $\mathrm{MeOH}(1 \mathrm{~mL})$. The stirring was continued in dark for another $1 \mathrm{~h}$. The reaction mixture was poured into 100 $\mathrm{mL}$ of ice-cold water containing a few drops of pyridine. The orange-yellow precipitate was filtered, washed with water, and dried. The product was purified on a silica gel coloumn chromatography.

Table S1. ${ }^{1} \mathrm{H}$ NMR for $2-\mathrm{X}-\mathrm{BnCo}(\mathrm{dmgH})_{2} \mathrm{Py}$ at room temperature on a JEOL JNM LAMBDA 400 FT NMR Spectrometer (at $400 \mathrm{MHz}$ ).

\begin{tabular}{|c|c|c|c|c|c|c|c|c|}
\hline \multirow[t]{2}{*}{$\mathrm{X}$} & \multirow[t]{2}{*}{$\mathrm{O}-\mathrm{H} \cdots \mathrm{O}$} & \multirow[t]{2}{*}{$\mathrm{CH}_{2}-\mathrm{Co}$} & \multirow[t]{2}{*}{$\mathrm{dmgH}$} & \multicolumn{3}{|c|}{ Pyridine } & \multirow[t]{2}{*}{ Aromatic } & \multirow{2}{*}{$\begin{array}{c}\text { Yield } \\
\%\end{array}$} \\
\hline & & & & $\alpha(d)$ & $\beta(t)$ & $\gamma(\mathrm{t})$ & & \\
\hline $\mathrm{H}$ & 18.30 & 2.85 & 1.95 & $\begin{array}{l}8.54 \\
(5.2)\end{array}$ & $\begin{array}{l}7.27 \\
(7.5)\end{array}$ & $\begin{array}{l}7.68 \\
(7.2)\end{array}$ & $6.97-7.02(\mathrm{~m}), 7.10-7.13(\mathrm{~m})$ & 70 \\
\hline $\mathrm{CH}_{3}{ }^{\mathrm{a}}$ & 18.37 & 2.92 & 1.93 & $\begin{array}{l}8.52 \\
(5.3)\end{array}$ & $\begin{array}{l}7.26 \\
(7.6)\end{array}$ & $\begin{array}{l}7.65 \\
(7.4)\end{array}$ & $6.79(\mathrm{~d}), 6.86(\mathrm{~d}), 7.03(\mathrm{~m})$ & 62 \\
\hline $\mathrm{Br}$ & 18.26 & 2.96 & 2.02 & $\begin{array}{l}8.53 \\
(5.2)\end{array}$ & $\begin{array}{l}7.27 \\
(7.6)\end{array}$ & $\begin{array}{l}7.68 \\
(7.5)\end{array}$ & $\begin{array}{l}\text { 6.96-6.98(m), 7.04-7.06(m), } \\
7.26(\mathrm{~d})\end{array}$ & 53 \\
\hline 2-Nap & 18.11 & 3.04 & 1.91 & $\begin{array}{l}8.66 \\
(5.5)\end{array}$ & $\mathrm{b}$ & $\begin{array}{l}7.73 \\
(7.5)\end{array}$ & $\begin{array}{l}\text { 5.88(s), 7.63(d), 7.36-7.22(m), } \\
7.94(\mathrm{t}), 8.02(\mathrm{~d}), 8.24(\mathrm{t})\end{array}$ & 58 \\
\hline $\mathrm{Br}^{\mathrm{c}}$ & 18.51 & 3.28 & 2.03 & $\begin{array}{l}8.70 \\
(5.2)\end{array}$ & $\mathrm{b}$ & $\begin{array}{l}7.75 \\
(7.7)\end{array}$ & $\begin{array}{l}\text { 6.93-7.05(m), 7.16-7.22(m) } \\
7.32-7.38(\mathrm{~m})\end{array}$ & 42 \\
\hline
\end{tabular}


Table S2. Coalescence temperature (Tc) and $\Delta \delta$ (at the lowest temp)

\begin{tabular}{|c|c|c|c|c|}
\hline \multicolumn{5}{|c|}{$\mathrm{CH}_{2} \mathrm{Co}$} \\
\hline & $\begin{array}{c}\mathbf{2 - B r} \\
(\mathbf{d m g H})\end{array}$ & $\begin{array}{c}\text { 2-Napthyl } \\
(\mathbf{d m g H})\end{array}$ & $\begin{array}{c}\mathbf{2 - C H} \\
\mathbf{( d m g H})\end{array}$ & $\begin{array}{c}\mathbf{2 - B r} \\
(\mathbf{d m g H})(\mathbf{d p g H})\end{array}$ \\
\hline $\mathrm{Tc}\left({ }^{\circ} \mathrm{C}\right)$ & -14 & -30 & -45 & -12 \\
\hline$\Delta \delta(\mathrm{Hz})$ & 255.8 & 33 & 9.2 & 200 \\
\hline \multicolumn{5}{|c|}{$\mathrm{dmgH}-\mathrm{Me}$} \\
\hline $\mathrm{Tc}\left({ }^{\circ} \mathrm{C}\right)$ & -50 & -23 & -20 & - \\
\hline$\Delta \delta(\mathrm{Hz})$ & 13.6 & 90 & 73.5 & - \\
\hline
\end{tabular}

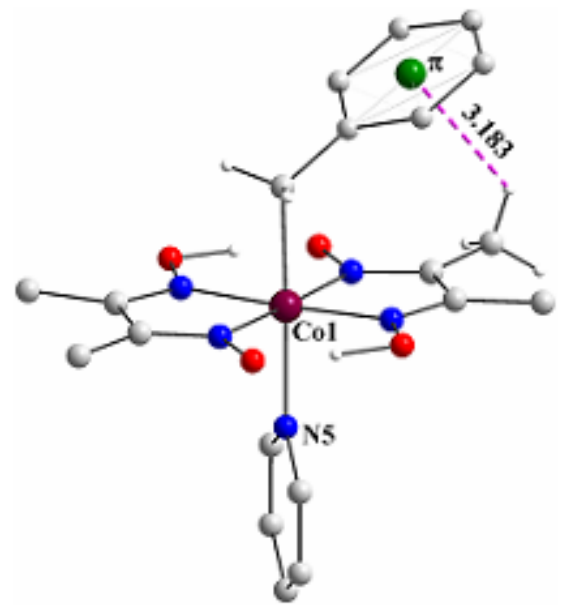

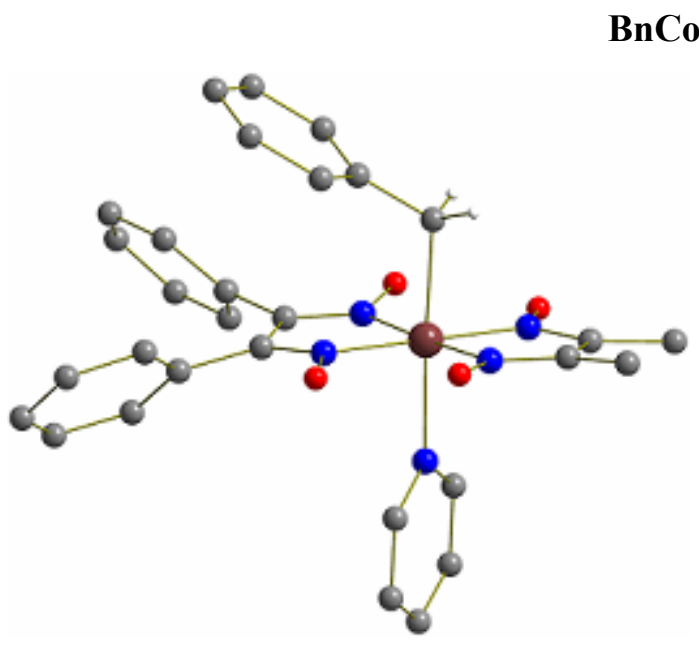

BnCo(dpgH)(dmgH)Py

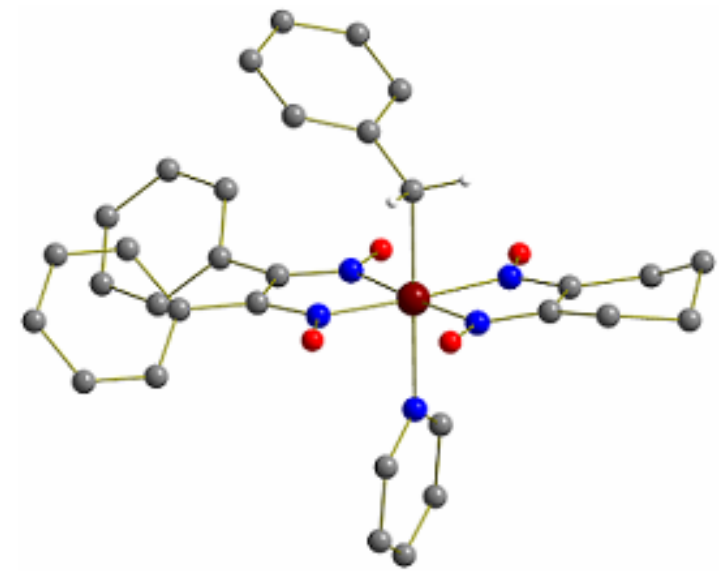

$\mathrm{BnCo}(\mathrm{dpgH})(\operatorname{chgH}) \mathrm{Py}^{2}$ 

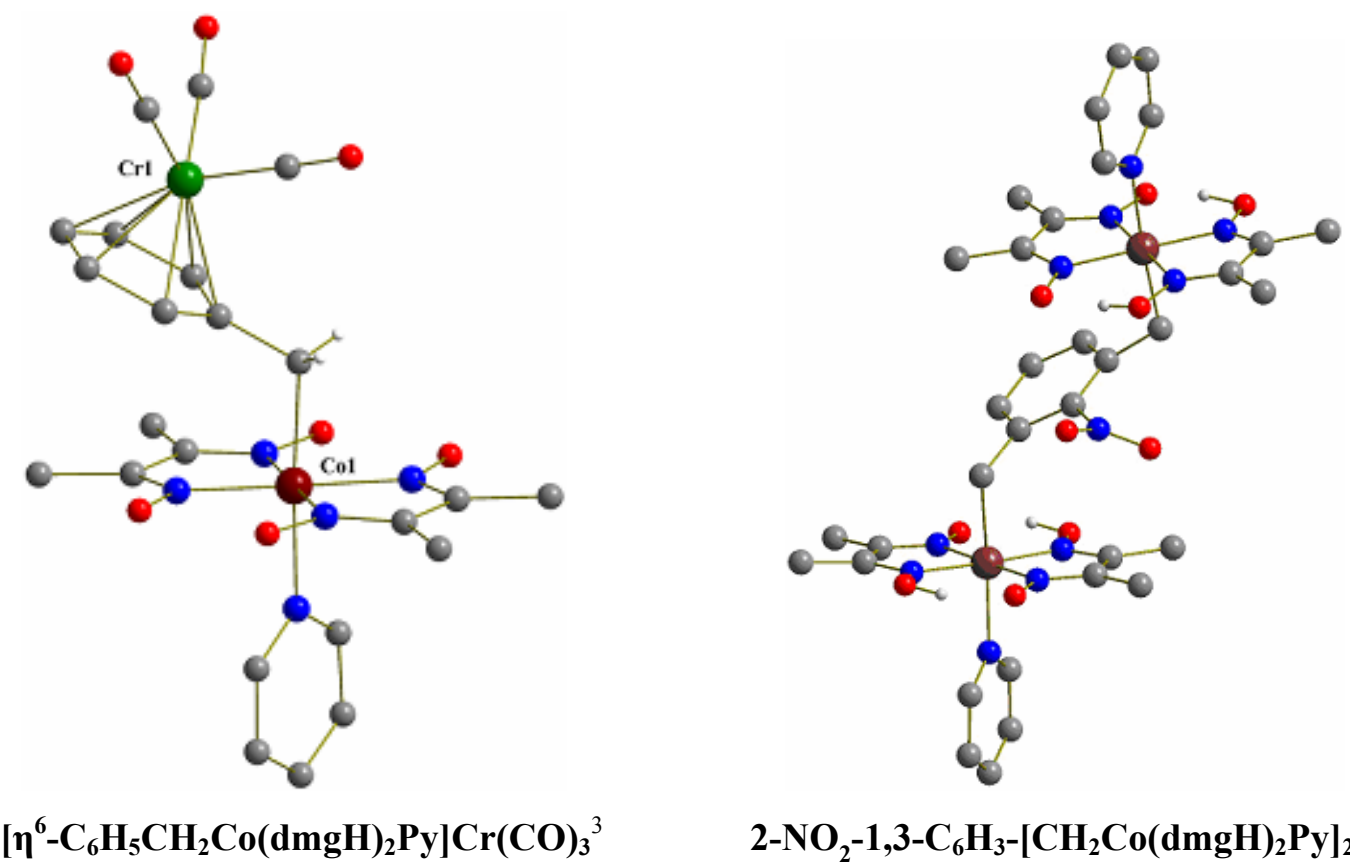

$2-\mathrm{NO}_{2}-1,3-\mathrm{C}_{6} \mathrm{H}_{3}-\left[\mathrm{CH}_{2} \mathrm{Co}(\mathrm{dmgH})_{2} \mathrm{Py}_{2}{ }^{4}\right.$

(1) $\mathrm{PhCH}_{2} \mathrm{Co}(\mathrm{dmgH})_{2} \mathrm{~B}$, including $\mathrm{B}=\mathrm{Py}(\mathrm{N}$. Bresciani-Pahor, Randaccio, L. Zangrando. E. Acta Crystallogr. Part C 1988, 44, 2052); B = pyrrolidine (Tyrlik, S. K.; Lenstra, A. T. H.; Van Loock, J. F. J.; Geise, H. J.; Dommisse, R. A. Acta Crystallogr. Part C 1986, 42, 553, and B = azaFc (Cesario, M.; Giannotti, C.; Guilhem, J.; Zakrzewski, J. Acta Crystallogr. Part C 1992, 48, 798).

(2) Gupta, B. D.; Yamuna, R.; Singh, V.; Tiwari, U.; Barclay, T.; Cordes W. J. Organomet. Chem. 2001, 627, 80.

(3) Domingo, M. R.; Irving, A.; Liao, Y.; Moss, J. R.; Nash, A. J. Orgnomet. Chem., 1993, 443, 232.

(4) Vijaikanth, V.; Gupta, B. D.; Mandal D.; Shekhar, S. Organometallics 2005, 24, 4454.

Figure S1. Orientation of Benzyl groups in representative Crystal Structures. (All cobaloximes with axial benzyl group lies over equatorial wing). 

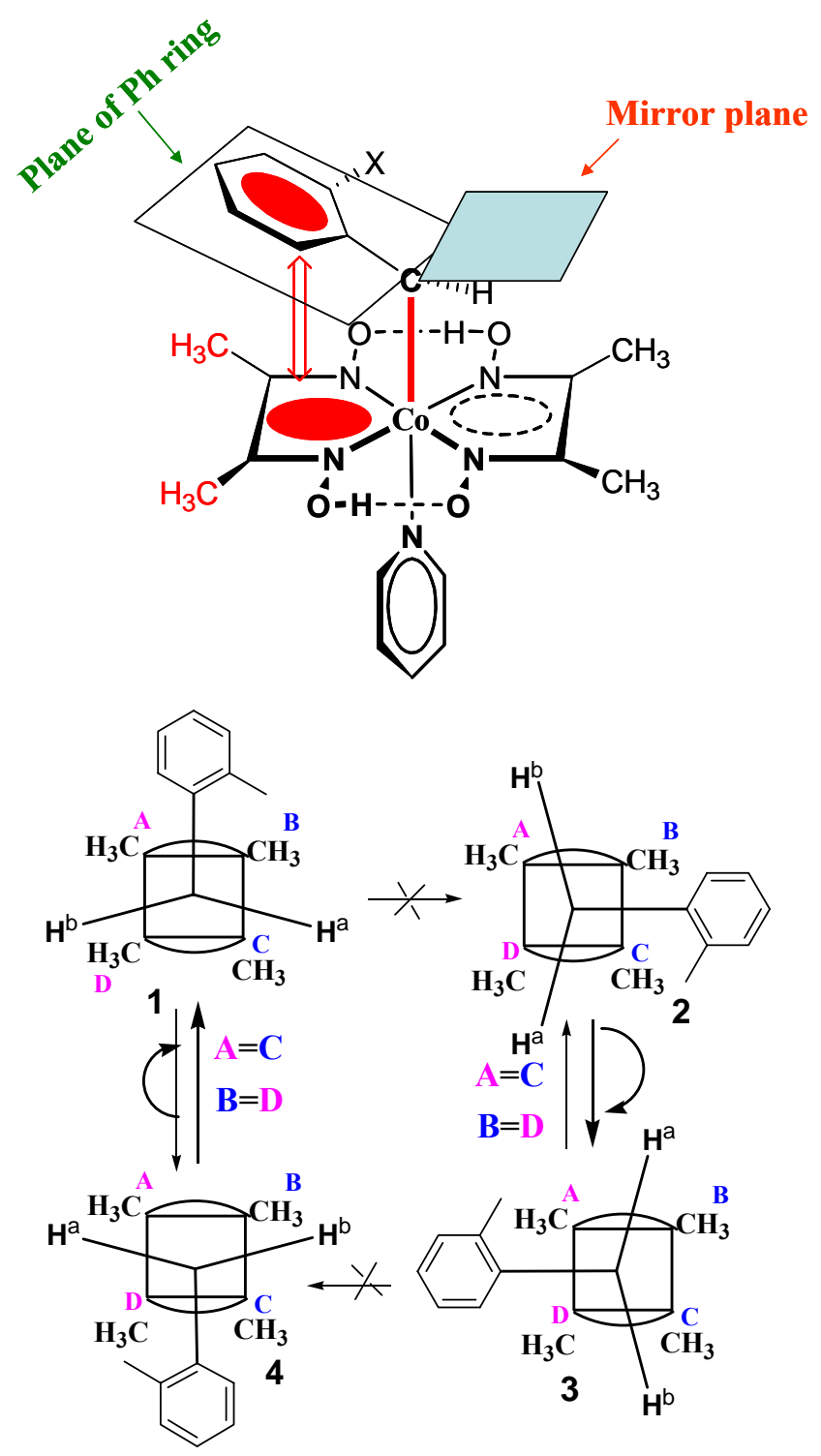

$\mathbf{A}=\mathbf{C}$ and $\mathbf{B}=\mathbf{D}$ only when there is free rotation about Co-C bond

Figure S2

\section{Possibilities:}

The arc is showing the $\mathrm{O}-\mathrm{H} \cdots \mathrm{O}$ bridging. Four isomers are possible due to the rapid rotation of Co- $\mathrm{C}$ bond provided $\mathrm{CH}_{2}$ is diastereotropic due to the restriction in the $\mathrm{C}-\mathrm{Ph}$ bond rotation. Since 1-4 and 2-3 are equivalent, two groups of diastereomers are formed. Each diastereomer will give two signals of $\mathrm{dmgH}$ methyl since $\mathrm{A}=\mathrm{C}$ and $\mathrm{B}=\mathrm{D}$. When Co-C bond rotation is restricted we can neglect the isomers 1 and 4 because the orientation of the benzyl is on $\mathrm{OH} \cdots \mathrm{O}$. The remaining two isomers may lead to $1: 1 \mathrm{dmgH}$ signals. 


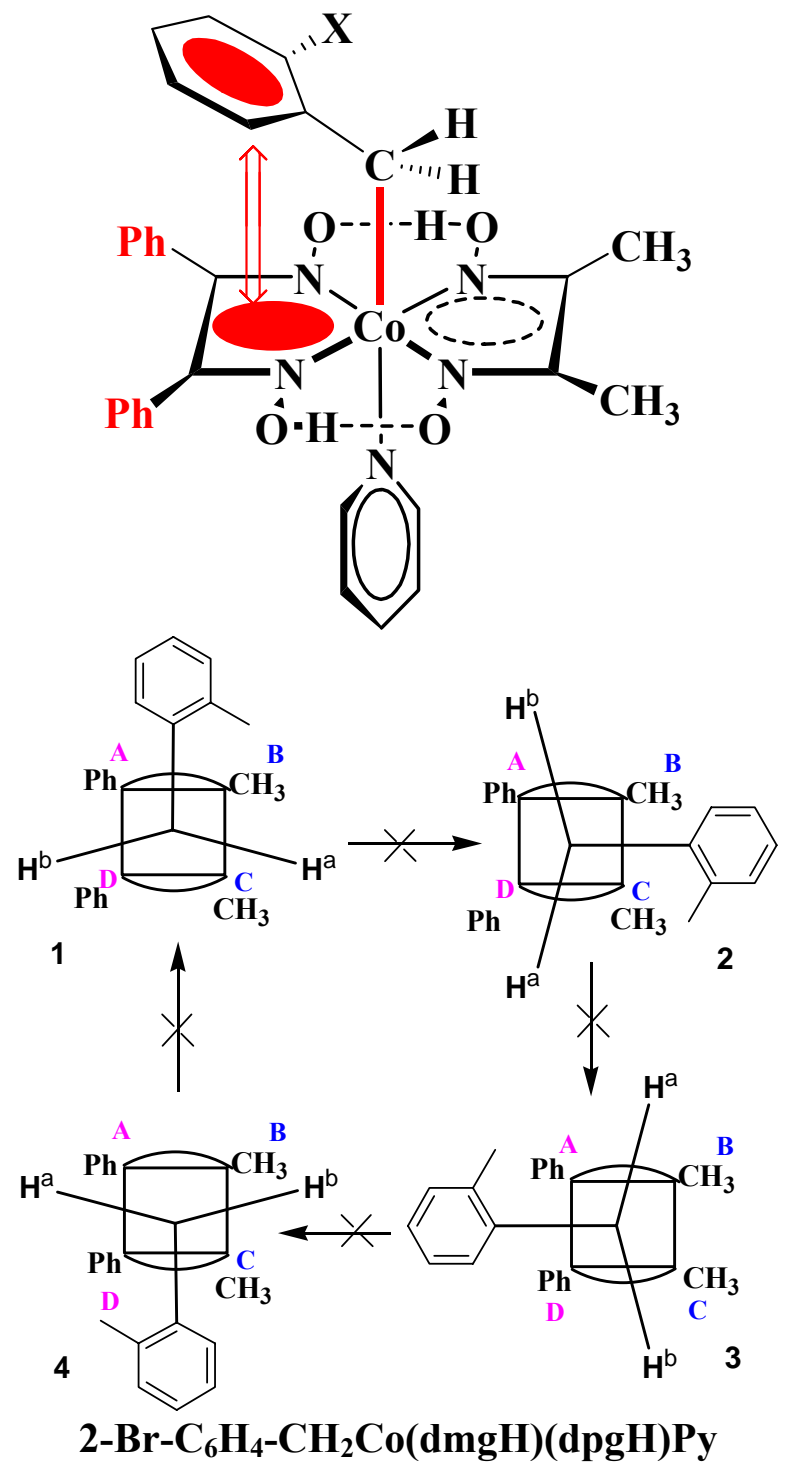

Figure S3

Rapid Co-C bond rotation will form 4 possible isomers of which no one is supper imposable and dmgH will give 4 sets of methyl signals (1:1:1:1). If Co-C bond partially or fully restricted then we can neglect the orientation over $\mathrm{O}-\mathrm{H} \cdots \mathrm{O}$ i.e. possibility of 1 and 4 . Remaining two isomers are possible but at the freezing temperature as per crystal structure only 3 will exist. Surprisingly, looking at the variable temperature ${ }^{1} \mathrm{H}$ NMR plots in figure 5 at $0{ }^{\circ} \mathrm{C}$ the $\operatorname{dmgH}$ shows the possible another isomer where the benzyl group is over $\mathrm{dmgH}$ wing. Is it due to the intermediate temperature of $\mathrm{Co}-\mathrm{C}$ bond restriction? 


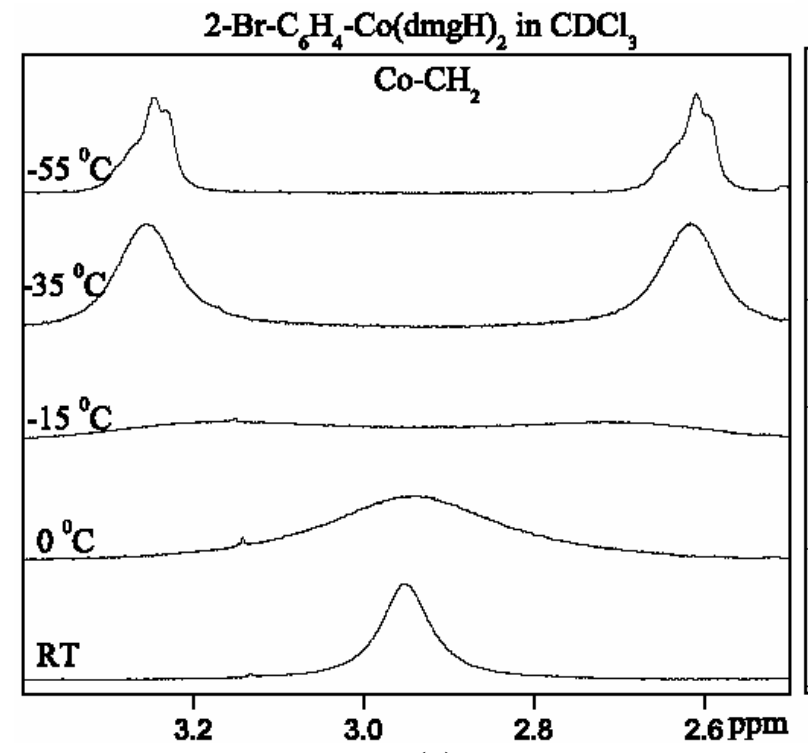

(a)

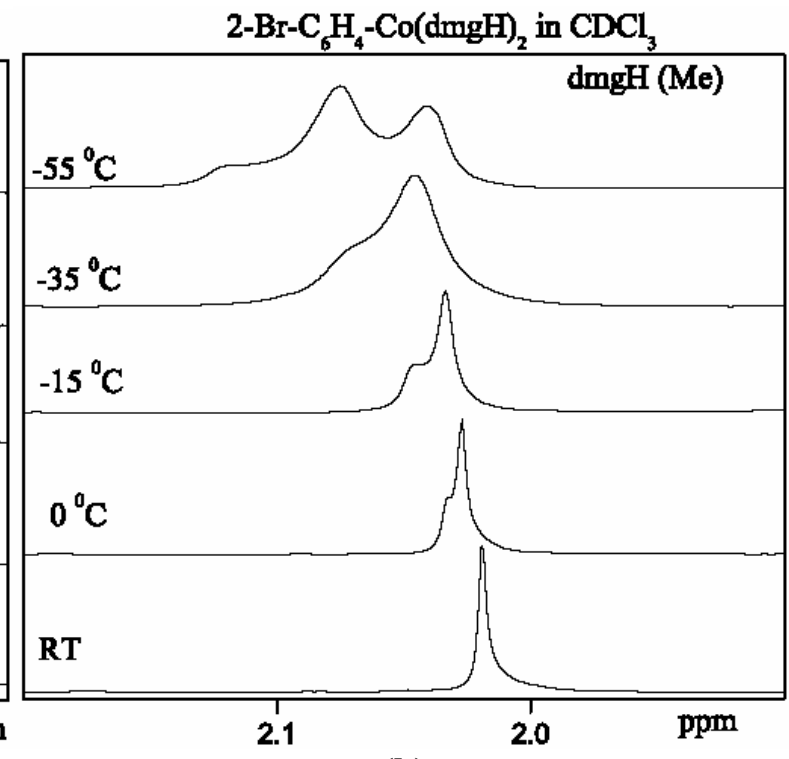

(b)

Figure S4. Variable temperature ${ }^{1} \mathrm{H}$ NMR spectra of the (a) $\mathrm{CH}_{2}-\mathrm{Co}$ and (b) dioxime (methyl) in 2- $\mathrm{Br}_{-} \mathrm{C}_{6} \mathrm{H}_{4} \mathrm{CH}_{2} \mathrm{Co}(\mathrm{dmgH})_{2} \mathrm{Py}$.

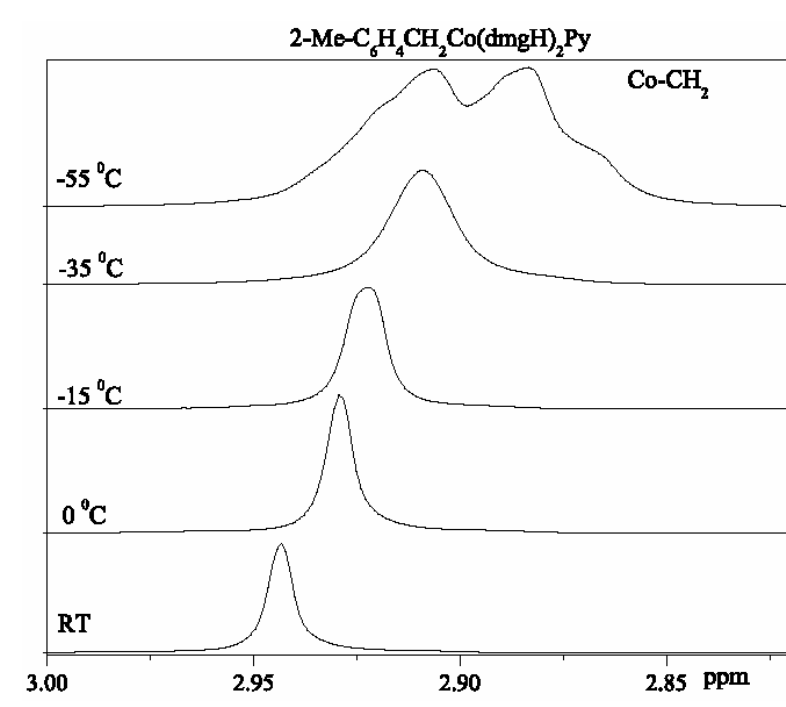

(a)

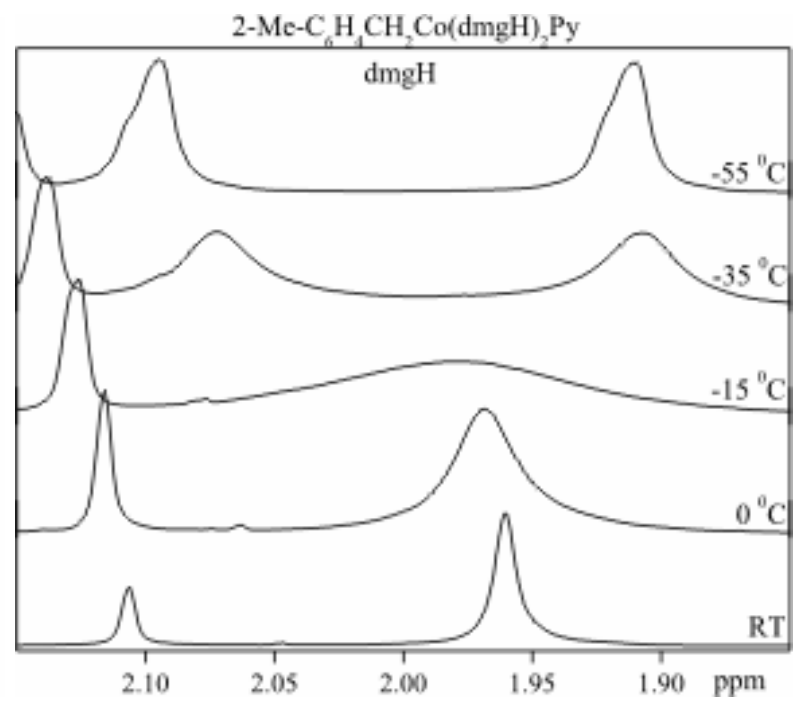

(b)

Figure S5. Variable temperature ${ }^{1} \mathrm{H}$ NMR spectra of the (a) $\mathrm{CH}_{2}-\mathrm{Co}$ and (b) dioxime (methyl) in $2-\mathrm{Me}-\mathrm{C}_{6} \mathrm{H}_{4}-\mathrm{CH}_{2} \mathrm{Co}(\mathrm{dmgH})_{2} \mathrm{Py}$. 


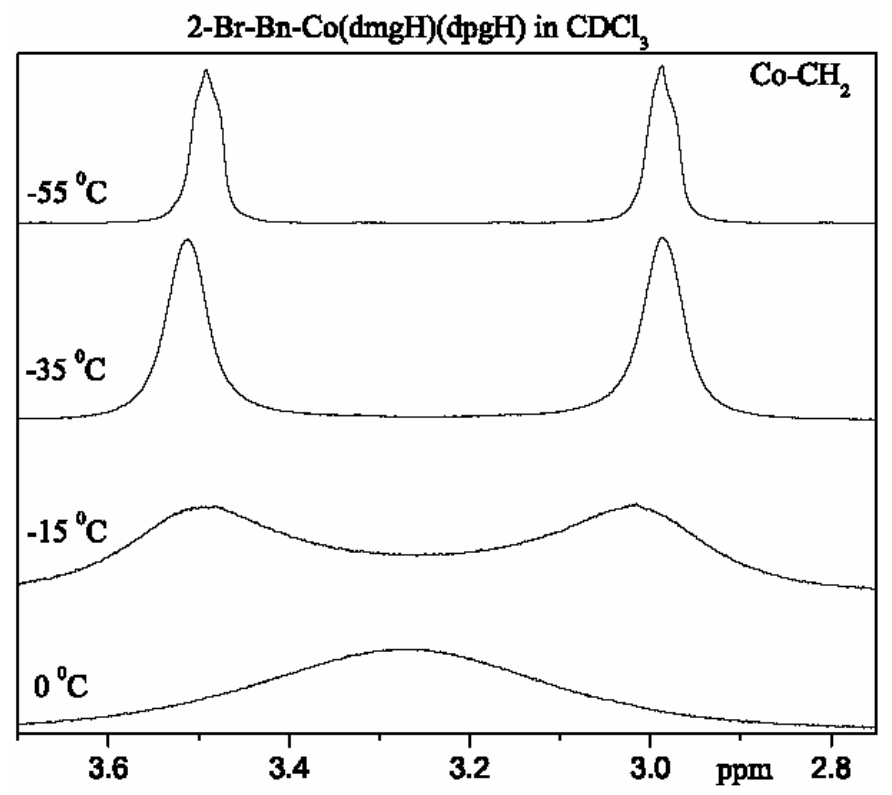

(a)

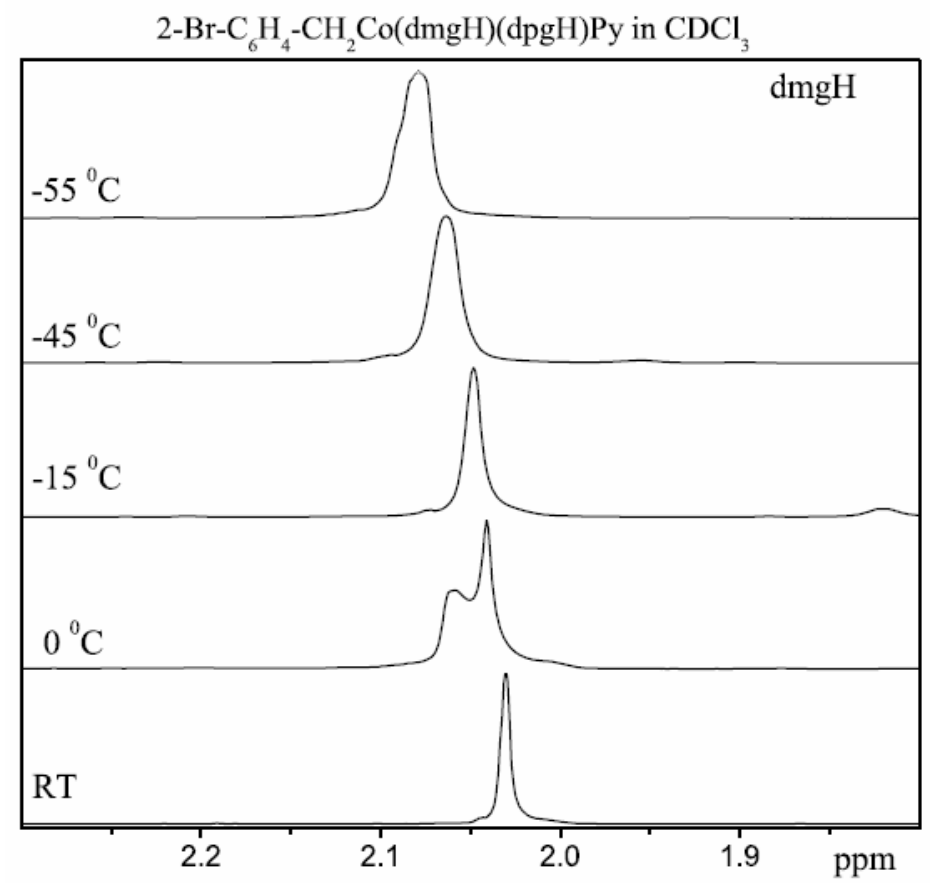

(b)

Figure S6. Variable temperature ${ }^{1} \mathrm{H}$ NMR spectra of the (a) $\mathrm{CH}_{2}-\mathrm{Co}$ and (b) dioxime (methyl) in $2-\mathrm{Br}_{-} \mathrm{C}_{6} \mathrm{H}_{4}-\mathrm{CH}_{2} \mathrm{Co}(\mathrm{dmgH})(\mathrm{dpgH}) \mathrm{Py}$. 
Table S3: Crystal Structure data

\begin{tabular}{|l|c|c|c|}
\hline & $\mathrm{BnCo}(\mathrm{dmgH})_{2} \mathrm{Py}^{\mathrm{a}}$ & $\begin{array}{c}2-\mathrm{CH}_{3}-\mathrm{C}_{6} \mathrm{H}_{4}- \\
\mathrm{CH}_{2} \mathrm{Co}(\mathrm{dmgH})_{2} \mathrm{Py}\end{array}$ & 2-Napthyl-Co(dmgH $)_{2} \mathrm{Py}$ \\
\hline $\mathrm{Co}-\mathrm{C}_{\mathrm{ax}}$ & $2.065(4)$ & $2.048(7)$ & $2.055(5)$ \\
\hline $\mathrm{Co}-\mathrm{N}_{\mathrm{ax}}$ & $2.056(3)$ & $2.060(7)$ & $2.047(4)$ \\
\hline $\mathrm{C}_{\mathrm{ax}}-\mathrm{Co}-\mathrm{N}_{\mathrm{ax}}$ & $177.1(1)$ & $176.1(3)$ & $177.7(2)$ \\
\hline $\mathrm{Co}-\mathrm{C}-\mathrm{C}$ & $116.7(2)$ & $118.1(4)$ & $115.1(4)$ \\
\hline$d$ & -0.0371 & -0.0208 & +0.0225 \\
\hline$\alpha(\operatorname{deg})$ & 1.86 & 1.74 & 6.90 \\
\hline$\tau(\mathrm{deg})$ & & 88.96 & 3.75 \\
\hline$\pi \cdots \pi(\AA)$ & 3.564 & 3.568 & $3.133(3.298)$ \\
\hline $\mathrm{C}-\mathrm{H}^{\cdots} \pi(\AA)$ & 3.183 & 3.122 & 3.134 \\
\hline
\end{tabular}

a Bresciani-Pahor, N.; Randaccio, L.; Zangrando, E. Acta Crystallogr., 1988, C44, 2052.

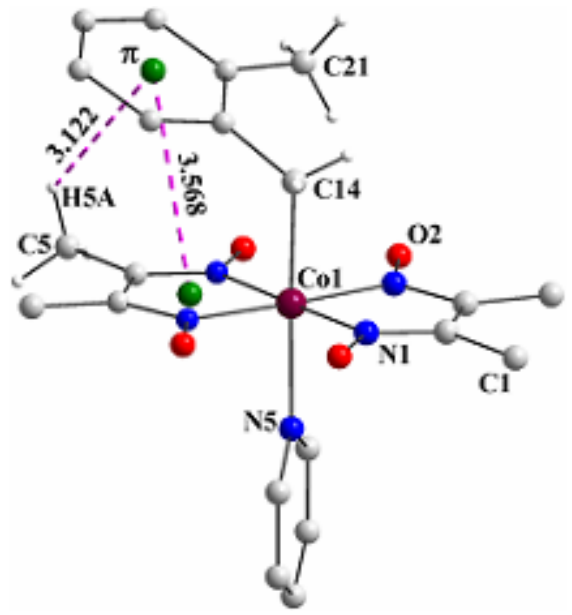

Figure S7. Crystal structure 2-Me- $\mathrm{C}_{6} \mathrm{H}_{4}-\mathrm{CH}_{2} \mathrm{Co}(\mathrm{dmgH})_{2} \mathrm{Py}$ and $\pi \cdots \pi / \mathrm{C}-\mathrm{H} \cdots \pi$ interactions. Methyl is away from the dioxime plane having no interaction.

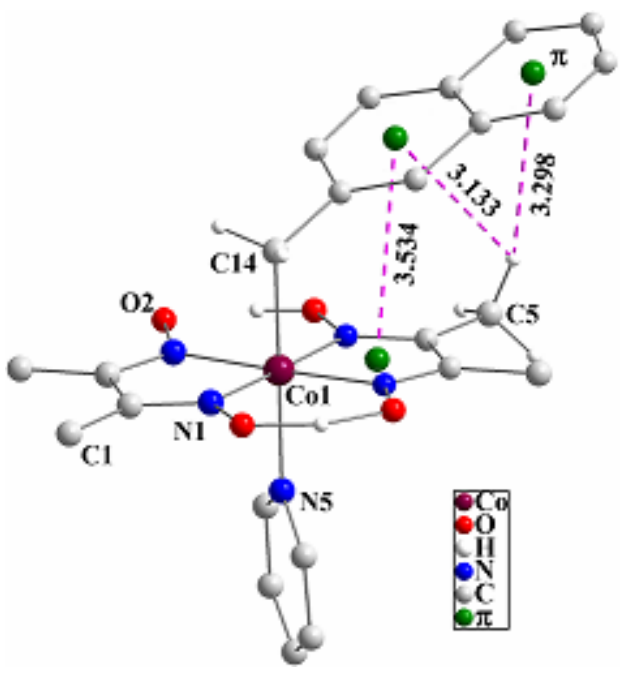

Figure S8. Crystal structure of 2-Napthyl-Co(dmgH $)_{2} \mathrm{Py}$ and $\pi \cdots \pi / \mathrm{C}-\mathrm{H} \cdots \pi$ interactions 

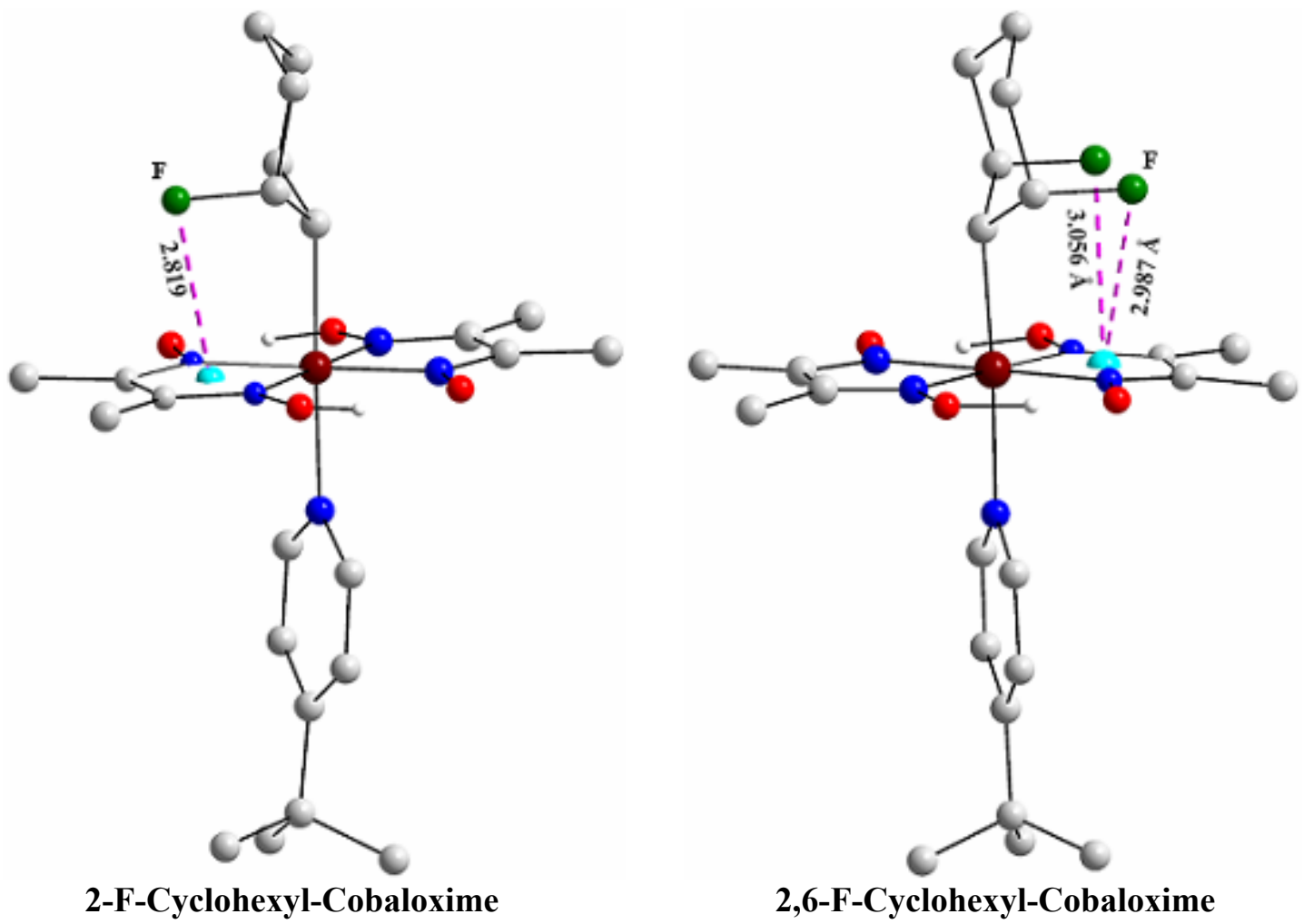

Figure S9. Reference 9 - it looks like fluorine is situated above the dioxime ring current having an interaction to influence the $\mathrm{Co}-\mathrm{C}$ bond restriction. 